University of Wollongong

Research Online

Australian Institute for Innovative Materials -

Papers

Australian Institute for Innovative Materials

$1-1-2019$

\title{
Patterning and process parameter effects in 3D suspension near-field electrospinning of nanoarrays
}

\author{
Alexander Nagle \\ University of Wollongong, arn734@uowmail.edu.au \\ Cormac Fay \\ University of Wollongong, cfay@uow.edu.au \\ Gordon G. Wallace \\ University of Wollongong, gwallace@uow.edu.au \\ Zhigang Xie \\ Deakin University \\ Xungai Wang \\ Deakin University
}

See next page for additional authors

Follow this and additional works at: https://ro.uow.edu.au/aiimpapers

Part of the Engineering Commons, and the Physical Sciences and Mathematics Commons

Research Online is the open access institutional repository for the University of Wollongong. For further information contact the UOW Library: research-pubs@uow.edu.au 


\title{
Patterning and process parameter effects in 3D suspension near-field electrospinning of nanoarrays
}

\author{
Abstract \\ The extracellular matrix (ECM) contains nanofibrous proteins and proteoglycans. Nanofabrication \\ methods have received growing interest in recent years as a means of recapitulating these elements \\ within the ECM. Near-field electrospinning (NFES) is a versatile fibre deposition method, capable of layer- \\ by-layer nano-fabrication. The maximum layer height is generally limited in layer-by-layer NFES as a \\ consequence of electrostatic effects of the polymer at the surface, due to residual charge and polymer \\ dielectric properties. This restricts the total volume achievable by layer-by-layer techniques. Surpassing \\ this restriction presents a complex challenge, leading to research innovations aimed at increasing \\ patterning precision, and achieving a translation from 2D to 3D additive nanofabrication. Here we \\ investigated a means of achieving this translation through the use of 3D electrode substrates. This was \\ addressed by in-house developed technology in which selective laser melt manufactured standing pillar \\ electrodes were combined with a direct suspension near-field electrospinning (SNFES) technique, which \\ implements an automated platform to manoeuvre the pillar electrodes around the emitter in order to \\ suspend fibres in the free space between the electrode support structures. In this study SNFES was used \\ in multiple operation modes, investigating the effects of varying process parameters, as well as pattern \\ variations on the suspended nanoarrays. Image analysis of the nanoarrays allowed for the assessment of \\ fibre directionality, isotropy, and diameter; identifying optimal settings to generate fibres for tissue \\ engineering applications.

\section{Disciplines} \\ Engineering | Physical Sciences and Mathematics

\section{Publication Details} \\ Nagle, A. R., Fay, C. D., Wallace, G. G., Xie, Z., Wang, X. \& Higgins, M. J. (2019). Patterning and process \\ parameter effects in 3D suspension near-field electrospinning of nanoarrays. Nanotechnology, 30 (49), \\ 495301-1-495301-13.
}

\section{Authors}

Alexander Nagle, Cormac Fay, Gordon G. Wallace, Zhigang Xie, Xungai Wang, and Michael J. Higgins 


\section{Patterning and Process Parameter Effects in 3D}

\section{Suspension Near-Field Electrospinning of Nanoarrays}

Alexander R. Nagle ${ }^{1}$, Cormac D. Fay ${ }^{1}$, Gordon G. Wallace ${ }^{1}$, Zhigang Xie ${ }^{2}$, Xungai Wang ${ }^{2}$, and Michael J. Higgins ${ }^{1 *}$

${ }^{1}$ ARC Centre of Excellence for Electromaterials Science, University of Wollongong, Innovation Campus, AIIM Facility, Squires Way, North Wollongong, New South Wales 2500, Australia.

${ }^{2}$ Institute for Frontier Materials, Deakin University, Geelong, Victoria 3216, Australia.

*Corresponding Author

Email:

mhiggins@uow.edu.au (Professor Michael. J. Higgins*) 


\begin{abstract}
The extracellular matrix (ECM) contains nanofibrous proteins and proteoglycans. Nanofabrication methods have received growing interest in recent years as means of recapitulating these elements within the ECM. Near-Field Electrospinning (NFES) is a versatile fibre deposition method, capable of layer-by-layer nano-fabrication. The maximum layer height generally limited in layer-by-layer NFES as a consequence of electrostatic effects of the polymer at the surface, due to residual charge and polymer dielectric properties. This restricts the total volume achievable by layer-by-layer techniques. To surpass this restriction presents a complex challenge, leading to research innovations aimed at increasing patterning precision, and achieving a translation from $2 \mathrm{D}$ to $3 \mathrm{D}$ additive nanofabrication.
\end{abstract}

Here we investigated a means of achieving this translation, through the use of $3 \mathrm{D}$ electrode substrates. This was addressed by previously developed in-house developed technology in which selective laser melt (SLM) manufactured standing pillar electrodes were combined with a direct Suspension Near-Field Electrospinning (SNFES) technique, which implements an automated platform to manoeuvre the pillar electrodes around the emitter in order to suspend fibers in the free space between the electrode support structures. In this study SNFES was used in multiple operation modes, investigating the effects of varying process parameters, as well as pattern variations, on the suspended nanoarrays. Image analysis of the nanoarrays allowed for the assessment of fiber directionality, isotropy, and diameter; identifying optimal settings to generate fibers for tissue engineering applications.

Keywords: near-field electrospinning, suspension, 3D electrode, nanoarray, nanofiber 


\section{Introduction}

With recent advances in tissue engineering, the possibility of successfully regenerating irreparably damaged tissue has become viable. Microtissue constructs (MTCs) operate as tissue substitutes for biomedical interventions such as tissue reconstruction ${ }^{1}$ as well as in vitro models to study cellular behaviour under treatment ${ }^{2,3}$. To develop MTCs, researchers have focused on imitating biological tissue structures, bioactivity, media flux and electrophysiology, which when combined promote the natural development of cells ${ }^{4}$. The native extracellular matrix (ECM) in human tissue is an amorphous 3D tissue embedded with fibrous networks of collagens, proteoglycans, fibronectin, laminin and peptides ${ }^{5-8}$.

The interconnective porosity of the ECM allows for the mobility of cells, nutrients and waste. The fibrous network component of the ECM, imparts physical and biochemical cues necessary for cell attachment, survival and development ${ }^{5}$. To mimic this tissue structure, MTC scaffold porosity, pore size, and interconnectivity must be controllably fabricated in 3D. A number of research findings have demonstrated that the structure and dimension of the environment dramatically affects cellular behaviour ${ }^{6,9,10}$. 3D cell culture mimics the physiological environment more effectively, resulting in improved cell survival and motility ${ }^{9,10}$ as compared to 2D environments. Improved cytocompatibility 11,12 and behavioral change ${ }^{13}$ has been shown in cell culture studies comparing submicron to supermicron fibers. In the case of neural ECM fiber orientation has been shown to guide neurite and neural cell alignment ${ }^{14} 15,16$ and migration along fibers through contact guidance in vitro ${ }^{17-20}$ as well as improve neural recovery in vivo ${ }^{21}$. These findings support the use of 3D structured nanofibrous components in MTCs. 
To emulate the protein/proteoglycan networks, nanofabrication techniques have received extensive technological investment and research. Solution based far-field electrospinning (FFES) is a technique capable of producing ultrafine polymer fibrous meshes, both aligned and randomly oriented. FFES can work using a wide variety of materials, has a simple set-up and can operate at room temperature ${ }^{22-24}$. Due to its similarity to the native tissue networks, the fibrous meshes produced by FFES have been investigated as an ECM mimicking structure through cell viability experiments ${ }^{12,25-31}$. However, conventional FFES lacks fine control of fiber deposition, where electrostatically driven bending instabilities lead FFES to produce disordered fibrous layers ${ }^{32}$. This lack of spatial control produces meshes with bulk pore sizes that are too narrow to allow for cell infiltration ${ }^{18}$.

Within the last ten years, electrospinning technology has advanced, allowing the direct patterning of nanofibers. Typically termed near-field electrospinning (NFES) or electrohydrodynamic direct writing ${ }^{33}$, these technologies have allowed directly written nanofibrous structures to be generated in designed patterns. This is achieved by reducing the working distance and applied voltage, precluding the jetting instability inherent to FFES. In this regime the control of fiber placement and morphology is achieved ${ }^{34}$. NFES can generate 3D structures by direct layer-by-layer deposition ${ }^{35-40}$ and this technique has allowed for the fabrication of 3D microfiber scaffolds for cell culture research in a number of studies $41-43$.

In our previous work, we developed a suspension near-field electrospinning (SNFES) instrument, which enabled fabrication of suspended fibrous nanoarrays between the pillars of $3 \mathrm{D}$ electrodes ${ }^{44}$. In this study, we investigate the effects of varying process parameters, such as speed, working distance, tip voltage, iteration and pattern variation, on 3D fiber arrays produced by the SNFES. The outcomes were measured by a number of metrics, for example, fiber array orientation and diameter distribution. 
In particular, the patterning results show that the SNFES method is effective in the production of multiple array patterns.

\section{Materials and Methods}

\subsection{Materials}

\subsubsection{Materials}

Titanium Grade 5 (Ti 6Al-4V) powder (spherical particle size $<63 \mu \mathrm{m}$ ) was purchased from TLS Technik GmbH (Bitterfeld, Germany). Poly(ethylene oxide) (PEO) powder (average $\mathrm{M}_{\mathrm{v}}$ : 200,000) was purchased from Sigma-Aldrich Co. (Sydney, Australia). PEO was dissolved at various concentrations $(8,11,14,17,20 \mathrm{wt} \%)$ in water/ethanol (3:2) using a magnetic stirring bar for $1 \mathrm{hr}$ to reach homogeneity.

\subsubsection{Apparatus}

Figure 1 (A-I) presents a schematic of the instrument assembly. Two linear motorised stages (ALSQ150B, Zaber) were overlaid perpendicular to each other (Figure 1 (B), (D)) to enable X-Y directional movement of the platform. A third stage (Figure $1(\mathrm{G})$ ) held a syringe pump (UMP3, World Precision Instruments Inc.) (Figure $1(\mathrm{~F})$ ) above the working area, moving vertically in the Z-axis. The stages, the syringe pump and the high voltage power supply were controlled by in-house developed software using the Qt software development kit (The Qt Company, Norway), written in C++ for high speed command execution. To allow for repeatable experiment operations and compatibility with 3D slicer programs, the stage system command line interface was programmed with an inline translator to enable the use of standard G-code. This system is described in greater detail in previous work ${ }^{44}$. 
A $1 \mathrm{~mL}$ syringe was mounted on the syringe pump, with a blunt tipped stainless steel needle $(0.25 \mathrm{~mm}$ inner diameter, Gauge 25). The needle was locked in place by a drill chuck, and grounded via ring connector. An in-house developed automatic cleaning device was placed beneath the tip. In the event of the tip moving within range of the cleaning paddles, a limit switch is triggered by the $\mathrm{Z}$ stage, and two servos compress the paddles on the tip. The tip is then retracted, drawing any residue from the emitter. Prolonged operation was achieved by periodic cleaning, preventing solution drying at the outlet and allowed the system to remain closed during operation. The NFES system was placed within a transparent acrylic enclosure for safety as well as to enable humidity control via a dehumidified compressed air line. All experiments were conducted at low humidity (ca. $19 \% \mathrm{RH}$ ), to prevent instabilities in NFES deposition. Humidity was measured using a temperature and humidity data logger (XC0424).

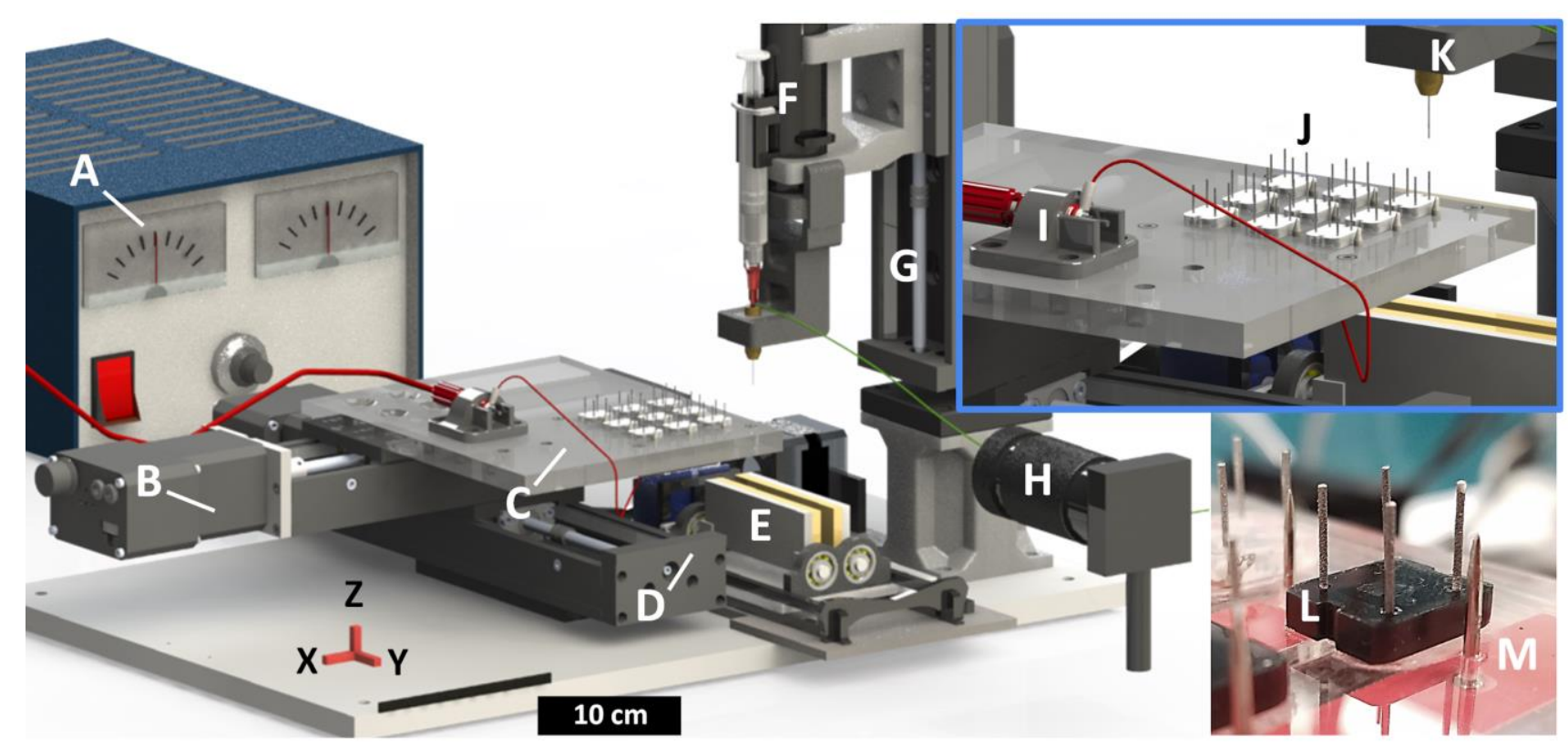

Figure 1. System Schematic. (A) High Voltage Power Supply; (B) X-Stage; (C) Platform; (D) YStage; (E) Automatic Cleaning Device; (F) Syringe pump; (G) Z-Stage; (H) Camera; (I) High voltage connection point; (J) Electrode array; (K) Blunt tipped Needle. (L) 4-pillar electrode with black cap mounted in the platform. (M) Initiation pin. 


\subsubsection{Electrode fabrication and mounting}

The titanium electrodes were manufactured using a SLM-50 (Realiser) from grade 5 titanium powder (Ti 6Al-4V). Figure 1 (L) shows the 4 pillar electrode embedded in the system platform. Electrode pillars are $7.07 \mathrm{~mm}$ separated along the horizontal/vertical and $10 \mathrm{~mm}$ along the diagonal. Insulating acrylic covers were attached over the base of the electrodes to ensure that the electric field lines emanated purely from electrode pillars to the syringe tip (Figure 1 (L)). The electrode design consisted of four vertical pillars, $700 \mu \mathrm{m}$ in diameter, $10 \mathrm{~mm}$ tall on an $\mathrm{x}$-shaped base, with two connection pins beneath the base (see Figure 2 (a)). On the X stage, an acrylic platform was attached to position the collecting electrodes (Figure $1(\mathrm{C}, \mathrm{J})$ ). The connection pins were plugged into three female 40 way IDC line socket connectors, firmly positioning the electrodes. The PCB header connected to a high voltage connection point (Figure 1 (I)) which led to a positive bias high voltage power supply (ES30, Gamma HV) (Figure 1 (A)).

\subsection{Suspended Nanoarray Fabrication}

\subsubsection{Parameter Variation Study}

PEO was chosen to test this suspension NFES method as this polymer has been extensively used in testing novel electrospinning methods, due to its simple water solubility. This eliminates the requirement of more volatile solvents typically applied with other polymers, reducing the risk of chemical burn to the user, and simplifying the procedure. When using highly volatile solvents the evaporation rate must be decreased by adding a co-solvent to avoid excessive drying. This solution's relatively low evaporation rate allows for greater timeframes in which to operate before nozzle tip obstruction by dried polymer. This is beneficial to NFES operation, particularly in the iteration studies conducted herein. 
To investigate the effects of process parameters on fibre arrays, a raster $(R)$ inter-pin pattern was selected. The two upright working planes were parallel to the plane created by the pillars (see Figure 2) at a working distance ( $W D)$, defined as the minimum distance between the emitter tip surface and the surface of the target pillars. Within the working planes, the tip was raised in steps of $100 \mu \mathrm{m}$ in the Z-axis for each horizontal move across the $\mathrm{X}-\mathrm{Y}$ plane, creating a programmed raster pattern $7 \mathrm{~mm}$ wide, with a total height of $5 \mathrm{~mm}$.

To calibrate the position of the syringe tip to the pillars, the stage was moved to align one pillar below the tip, and the tip was lowered until contact with the pillar's apex. G-code script was then generated from this calibration using a spreadsheet program (Google Sheets). The solution extrusion rate was held constant $3 \mathrm{nl} . \mathrm{s}^{-1}$ to maintain the solution droplet. To better understand the patterning process, readers may refer to the Video 1 in the supplementary section.

Electrospinning requires that the jet be initiated, and this can be done in two established ways. The first applies a critical voltage, exerting a high electrostatic force on the droplet surface, which overcomes its surface tension. The second method exerts a mechanical initiation by means of disruption of the droplet surface ${ }^{45,46}$. Mechanical initiation was used in this study, as there is a greater risk of arcing at the low WD applied. In this technique, initiation involved contacting the solution droplet on the apex of a sharp pointed stainless steel tip, embedded in the surface of the platform (see Figure $1 \mathrm{M}$ ), immediately followed by the intended pattern. This was achieved automatically, following an initial manually handled calibration.

In order to determine the optimal parameters for PEO in SNFES, two orthogonal experiment groups were examined, investigating four treatment parameters. In the first group (T1) maximum speed $\left(S_{\max }\right)$ was varied while $W D$ was varied as described in table 1 . In this orthogonal group, $1.6 \mathrm{kV}$ was applied 
using a 14 wt\% PEO solution. In the second group (T2) the applied voltage and PEO solution concentration were varied as described in table 2 . A constant $S_{\max }$ of $50 \mathrm{~mm} \cdot \mathrm{s}^{-1}$ and a $W D$ of $1 \mathrm{~mm}$ were applied. For each unique setting, samples were reproduced three times or until a suitable number of successful samples were generated $(n \geq 4)$.

Table 1. Orthogonal sample grouping T1. Constants $1.6 \mathrm{kV}$ and $14 \mathrm{wt} \%$ PEO.

\begin{tabular}{|l|l|l|l|l|l|l|l|l|l|}
\hline$S_{\max }$ & 50 & 150 & 250 & 50 & 150 & 250 & 50 & 150 & 250 \\
\hline$W D$ & \multicolumn{3}{|c|}{1.5} & \multicolumn{3}{|c|}{2} \\
\hline
\end{tabular}

Table 2. Orthogonal sample grouping T2. Constants Smax of 50mm.s-1 and WD of $1 \mathrm{~mm}$

\begin{tabular}{|c|c|c|c|c|c|c|c|c|c|c|c|c|c|}
\hline Voltage & 1.2 & 1.6 & 2 & 1.6 & 1.2 & 1.4 & 1.6 & 1.8 & 2 & 1.6 & 1.2 & 1.6 & 2 \\
\hline Concentration & & 8 & & 11 & & & 14 & & & 17 & & 20 & \\
\hline
\end{tabular}

\subsubsection{Iteration Study}

To investigate the effects of multiple repeated patterning, a square iteration $(\mathrm{Si})$ pattern was applied (see Figure $2(\mathrm{~b}-\mathrm{c})$ ). This follows a square path around the four pillars for a certain number of laps, followed by a move upwards in the Z-axis. A total of nineteen sequential Z-axis positions were used, each separated by $0.1 \mathrm{~mm}$, resulting in a total height of $1.9 \mathrm{~mm}$. In addition, this study acts to stress test the technique, to ensure optimal automation over an extended time period. To achieve reproducibility, a semi-automatic process was necessary. This process involved a looping sequence of cleaning, to mitigate discontinuity of ejection from the droplet, which can be caused by the solution drying at the outlet as well as contamination of the tip by particulate accumulation (among other interruptive events). The cleaning process was followed by mechanical initiation and $S i$ patterning. This cleaning sequence was performed at regular intervals, multiple times proportional to the pattern iteration value. An example of the patterning operation is shown in supplementary Video 1 
The number of pattern iterations (i.e. laps, circuits) performed for every $\mathrm{Z}$ position was varied as a group of independent samples: of 1, 15, 60, 105 and 150 iterations. The applied voltage $(1.6 \mathrm{kV})$, and PEO concentration (14 wt $\%$ ) were held constant in these experiments. The solution extrusion rate was kept constant, i.e. $3 \mathrm{nl} . \mathrm{s}^{-1}$. The $W D$ was varied at 1 and $1.5 \mathrm{~mm}$, and samples were generated in quadruplicate for each unique setting $(n=4)$.

\subsubsection{Patterning Variation}

Fibers of PEO were suspended between electrode pillars using a variety of pattern types to investigate the versatility of the technique, and test the effect of these patterns on the array alignment, fibre density, diameter and morphology. Figure 2 (b) illustrates the toolpaths of these patterns. The first pattern is the previously described Si pattern. The second pattern denoted: \, creates a circuit lapping around two diagonal pins. The second pattern combines the \pattern and the Si pattern (denoted: \Si).The third pattern combines the $\backslash$ pattern followed by its counterpart / around alternate pins (denoted $\bigvee$ ). This pattern operates within distinct height regions for each pattern to avoid collision of the tip with the deposited structure. Each pattern was repeated 15 times (iterations) per Z position, moving a total height of $1.9 \mathrm{~mm}$. These patterns were performed at $S_{\max }$ of 50 and $250 \mathrm{~mm} . \mathrm{s}^{-1}$ in separate groups $(\mathrm{n}>4)$. The applied voltage $(1.6 \mathrm{kV}), W D(1 \mathrm{~mm})$ and PEO concentration $(14 \% \mathrm{wt})$ were held constant. Solution extrusion rate was maintained at $3 \mathrm{nl} . \mathrm{s}^{-1}$. 


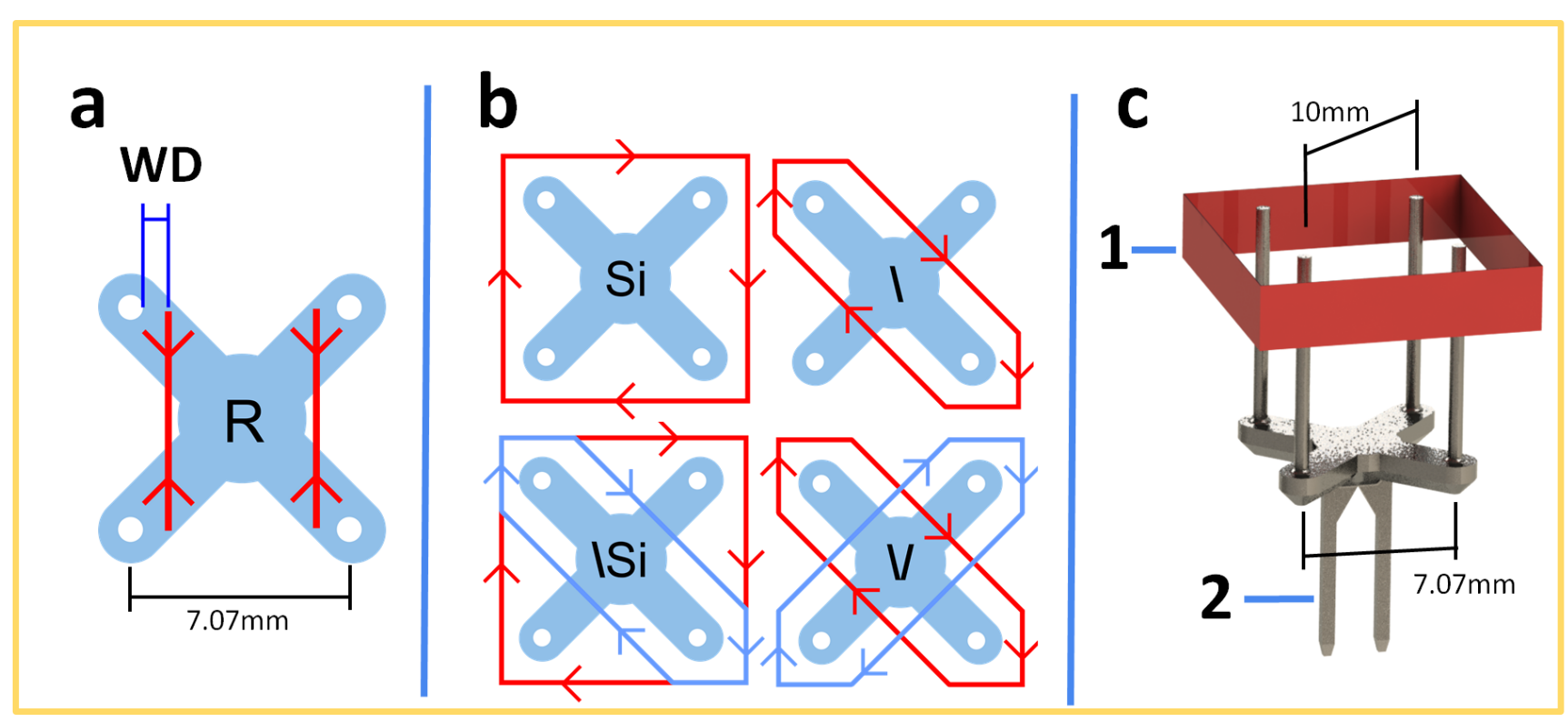

Figure 2. Pattern Variations. (a) R pattern tool-path (red) with working distance (Wd) shown. Each path moves back and forth at both positions. (b) Pattern tool-path variations with identifiers and routes (red and blue). (b) Pattern variations with identifiers and tool-paths (red and blue). (c) 3D CAD illustration of the Si pattern (1), showing the electrode connection pins (2).

\subsection{Characterisation}

\subsubsection{Rheological Analysis}

The polymer solutions of each concentration were analysed using a rheometer (ARG2, TA Instruments, Delaware, USA), measuring the viscosity as a function of shear rate, from 0.01 to $100 \mathrm{~s}^{-1}$ in a rotating cone and plate system.

\subsubsection{Light Microscopy}

3D fiber arrays were imaged by light microscopy (M205A, Leica Microsystems, Wetzlar, Germany) observing orthogonal perspectives of each array plane (see article ${ }^{44}$ for additional details). 


\subsubsection{Scanning Electron Microscopy}

The morphology of each nanoarray generated was investigated by scanning electron microscopy (SEM), with the exception of samples generated in section 2.2.3 Patterning Variation. Four pillar electrodes were mounted on stainless steel stubs by carbon tape, and SEM imaging was carried out on the entire electrode. SEM was performed using a JSM-7500FA (JEOL Ltd.) operating at $10 \mathrm{kV}$, and 5 mA. For each sample, a minimum of five high-magnification images $(\sim 2000 \times)$ were taken of fibres comprising the array $(\mathrm{n}>5)$.

\subsubsection{Image analysis}

Fiber arrays imaged by light microscopy were assessed for their order in terms of their distribution. This was quantified in terms of their normalised orientation distribution (OD), and estimated fibre number. The normalised OD is analogous to a probability density function of fiber orientation. Microscope images were converted to binary segmented images and semi-automatically analysed using 'Diameter J' ${ }^{47}$, a plugin of image processing software ImageJ (National Institutes of Health, USA). This plugin applies the OrientationJ module to determine the OD of the fibers as well as producing axially thinned representations of the fibre arrays ${ }^{48}$. Refer to supplementary figure for an illustration of this process. To allow the comparison of distributions, we calculated the preferred orientation, defined by the distribution centroid $\left(\phi_{c}\right)^{49}$ defined in equation 1 as:

$\phi_{C}=\frac{\sum_{\phi=\phi_{\min }}^{\phi \min ^{+180^{\circ}}}\left[(\phi-\phi \min ) \frac{I(\phi)+I(\phi-1)}{2}\right]}{\sum_{\phi=\phi}^{\phi \min ^{+180^{\circ}}\left[\frac{I(\phi)+I(\phi-1)}{2}\right]}}$

Where $\phi_{c}$ is the distribution centroid, $\phi$ is the angle, and $\phi_{\min }$ defines the starting position, $0^{\circ}$ in this case, and thus the range of 180 degrees of analysis. Furthermore, we define the distribution skew as $\phi_{\mathrm{s}}$ $=\phi_{\mathrm{p}}-\phi_{\mathrm{c}}$, where $\phi_{\mathrm{p}}$ is the maximum peak orientation obtained by peak analysis (i.e. global maximum) 
The greater the degree of $\phi_{\mathrm{s}}$ divergence from a symmetrical distribution the greater the likelihood the fiber OD is not a single population. All distribution fitting, centroid calculations and peak analysis was conducted using OriginPro 2015 software (OriginLab Corp.). The code for centroid calculation is shown in supplementary section 1 .

To indicate the degree of fibre alignment we describe the width of the distribution using the Orientation Index (OI $)^{47,49,50}$. The OI is defined as the number of degrees expanded from $\Phi_{\mathrm{c}}$ symmetrically in both clockwise and anticlockwise directions, which contains $50 \%$ of the total normalised frequencies (see Figure 3 (b) for an example). From this we can derive the intuitive Normalised Orientation Index $(\mathrm{NOI})$ : where $N O I=\frac{\left(90^{\circ}-O I\right)}{90^{\circ}} \times 100, N O I \in[0,100]$. The NOI values of $0 \%$ (binary peak, lattice) or $100 \%$ (single peak, array), indicate highly aligned fibres, whereas values c.a. $50 \%$ indicate a random fiber distribution.

To estimate the number of fibres per sample, the axially thinned images of each array, generated by DiameterJ, were sectioned by four horizontal lines equally spaced across each image. Each line's pixel profile was measured and the number of fibres crossing the line were counted. The four counts were averaged to give the approximate fibre number in each sample. From SEM micrographs the diameters of the fibers were measured using ImageJ tools. Measurements of fiber diameter were performed at ten random locations in each micrograph manually, with a minimum of five images per sample to investigate diameter variability. This method is similar to one used in the literature ${ }^{51}$.In several samples for the purposes of illustration SEM images are analysed using the DiameterJ function giving the frequency histogram of the diameter in the image.

To estimate the number of fiber intersections, microscopy images of the arrays were analysed. Images were prepared by threshold binarising and conversion to axially thinned fibers by ImageJ functions. 
Branch pruning by length was performed via a Beanshell script running in ImageJ, using a threshold of 150 pixels in length which equals ca. $0.41 \mathrm{~mm}$, removing smaller fibrils splaying from the main fibers as well as image artifacts of skeletonizing the binary image. This was then followed by the analyse skeleton function of ImageJ, outputting intersection number. This method was applied for all samples using the raster type pattern.

\subsubsection{Statistical analysis}

To evaluate the independent distributions, two-sample t test was used to determine the significant difference between groups. Mean fibre number (Section 2.2.3 Patterning Variation) were analysed to determine significance of variation between means. Means were subjected to the Shapiro-Wilk normality test, Levene's test of homogeneity of variance, and univariate ANOVA with the GamesHowell test. All calculations were performed using SPSS Statistics 23 (IBM, USA). 


\section{Results and Discussion}

\subsection{Effects of process parameters on nanoarrays}

In electrospinning there are many effective parameters of control, which can be broadly grouped into three categories: environmental, solution and process parameters ${ }^{45,52,53}$. Additionally, certain regimes of electrospinning can be classified: spraying ${ }^{54}$, beading ${ }^{34,55}$, continuous, and intermittent ${ }^{56}$. These regimes define the effective parameter domains of continuous electrospinning. These parameters are interdependent when predicting a continuous electrospinning parameter group. In this study, relative humidity and flow rate were fixed, while varying the most readily controllable processes in two treatment groups, T1 and T2 (see Tables 1 and 2). The parameter range was constrained by the physical system (max speed, polymer concentration), as well as by dielectric breakdown (voltage, working distance). Air begins to break down when electric field exceeds approximately $3 \mathrm{kV} . \mathrm{mm}^{-1}$.NFES operation discontinues as the arcing causes rapid drying, and solidification of polymer at the emitter outlet, as well as disrupting communication with system instruments

The minimum integer working distance which could be accurately applied was $1 \mathrm{~mm}$, given the accuracy of calibration and the varying degree of angular divergence of both electrode and emitter from upright. The maximum working distance was defined by what had previously been found to be effective ${ }^{44}$, where the working distance is typically no greater than $10 \mathrm{~mm}$ for solution based NFES. Within these experiments voltages above $2 \mathrm{kV}$ using a working distance of $1 \mathrm{~mm}$ were found to commonly result in dielectric breakdown. This defined the upper limit of voltage operation. The lower voltage limit was defined by the success in coherent jetting, and this varied for differing concentrations. $1.2 \mathrm{kV}$ was found to be the minimum effective voltage across all sample concentrations used, and this defined the domain of effective operation for applied voltages, i.e. between 1.2 and $2 \mathrm{kV}$. 


\subsubsection{Effect of process parameters on nanoarray distribution}

The R pattern would ideally produce highly aligned, parallel, equally spaced fibres in a precise number according to the design. We can quantitatively compare the precision between treatments in terms of the NOI (i.e. variance) and the global maximum peak information of each OD. The ideal distribution would have a global maximum peak at $90^{\circ}$ (relative to the pillars), and an NOI of $100 \%$. The first treatment group (T1) varied the working distance as well as the $S_{\max }$ in a 3 level orthogonal experiment. The second group (T2) varied the voltage and the PEO concentration in a separate 3 level orthogonal experiment. Figure 3 (a) shows an example of a suspended array and its corresponding OD (Figure 3 (b)). Lorentz fitting of this OD showed a centre point of $87.2^{\circ} \pm 0.9$, with full width at half maximum $\left(\mathrm{FWHM}\right.$ ) value of $24.2^{\circ} \pm 3.1$. This example is accurate (ca. $90^{\circ}$ orientated), yet imprecise (broad OD distribution, NOI $=80 \%$ ).
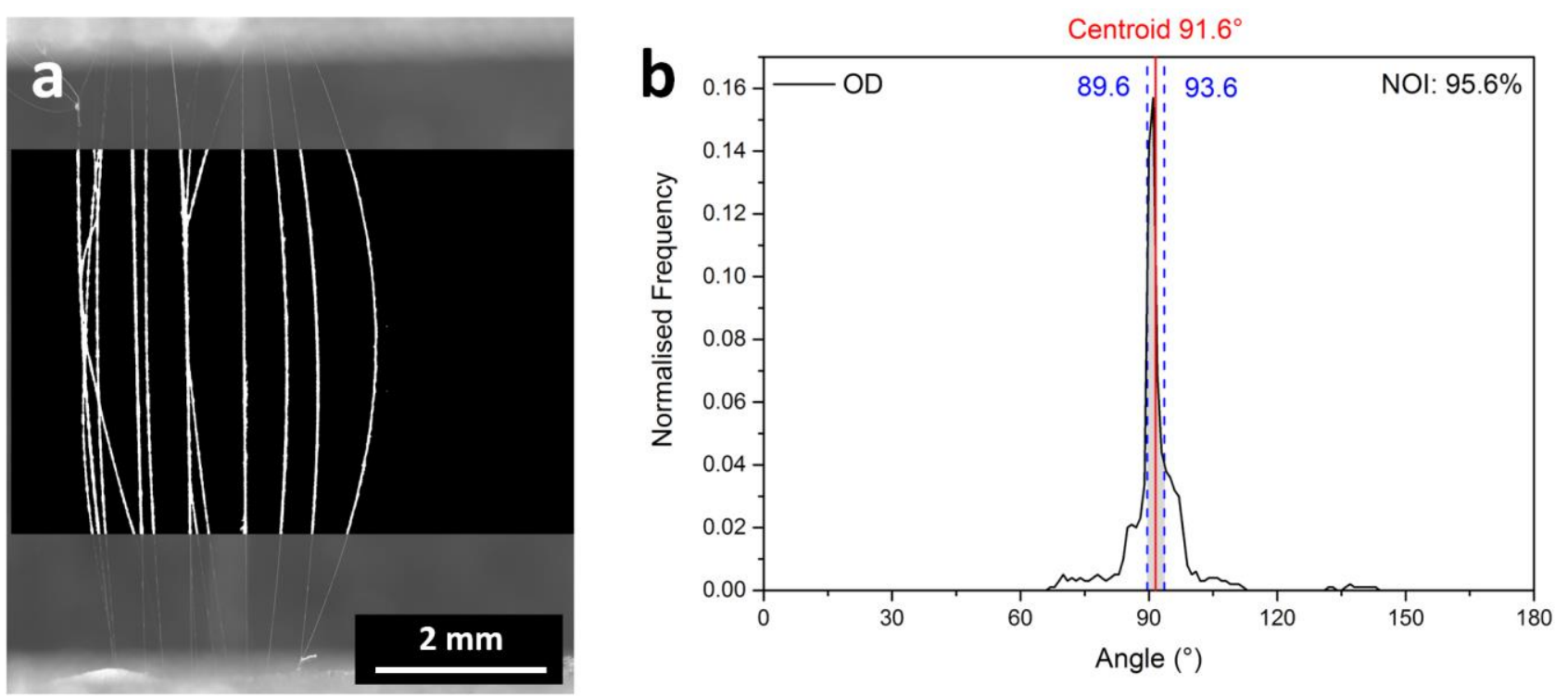

Figure 3. R patterned nanofibrous arrays. (a) Microscopy image of PEO fibre array generated using the R pattern. The overlay shows a binary segmented view of the fibres, generated by image processing. (b) The extracted OD for image A, with red line indicating a centroid of $91.6^{\circ}$. Dashed blue lines indicate the boundaries of the OI region (grey). The OI was calculated from the OD without the use of fitting distribution models. 
Figure 4 (a) shows a box plot of global maximum angle (red), and NOI (blue) for each OD as a function of process parameters $S_{\max }$ and $W D$. The distributions of the maxima are bounded in the region of $90^{\circ}$ $\pm 5^{\circ}$, while NOI is above $80 \%$ with a few exceptions. This accuracy and precision information indicates $90^{\circ}$ aligned fibre arrays, and in particular a WD of $2 \mathrm{~mm}$ and most accurate global orientations with correspondingly high NOI. In comparison, Figure 4 (b) shows a greater variability in accuracy and precision in orientation with changing voltage and concentration. The NOI often reaches below $80 \%$ with the greatest decline in samples produced using $20 \mathrm{wt} \% \mathrm{PEO}$, indicating poor array alignment. The global maximum peaks deviate from $90^{\circ}$ in the sample generated using concentrations of $20 \%$ wt and particularly in $1.2 \mathrm{kV}, 8 \mathrm{wt} \%$ samples.

The minimum working distance used here of $1 \mathrm{~mm}$, was restricted by dielectric breakdown arcing effects. This may be overcome as in 2D NFES, where working distance as low as ca. $0.2 \mathrm{~mm}$ can be achieved, by reducing voltage and by working within an enabling solution parameter range. If this intervention does not readily prevent dielectric breakdown, it may be necessary to work in a sealed controlled environment to manipulate the dielectric strength of air. This can be achieved in a number of ways using the principles of Paschen's Law. In air dielectric strength becomes exponentially higher as electrodes approach contact, higher pressure leads to an increase in the dielectric strength and at extremely low pressures the dielectric strength is much greater. Reducing absolute humidity would also improve the dielectric strength of air. These untested interventions present a number of challenges in their implementation for SNFES, and may have unpredictable effects which will need to be further investigated. 

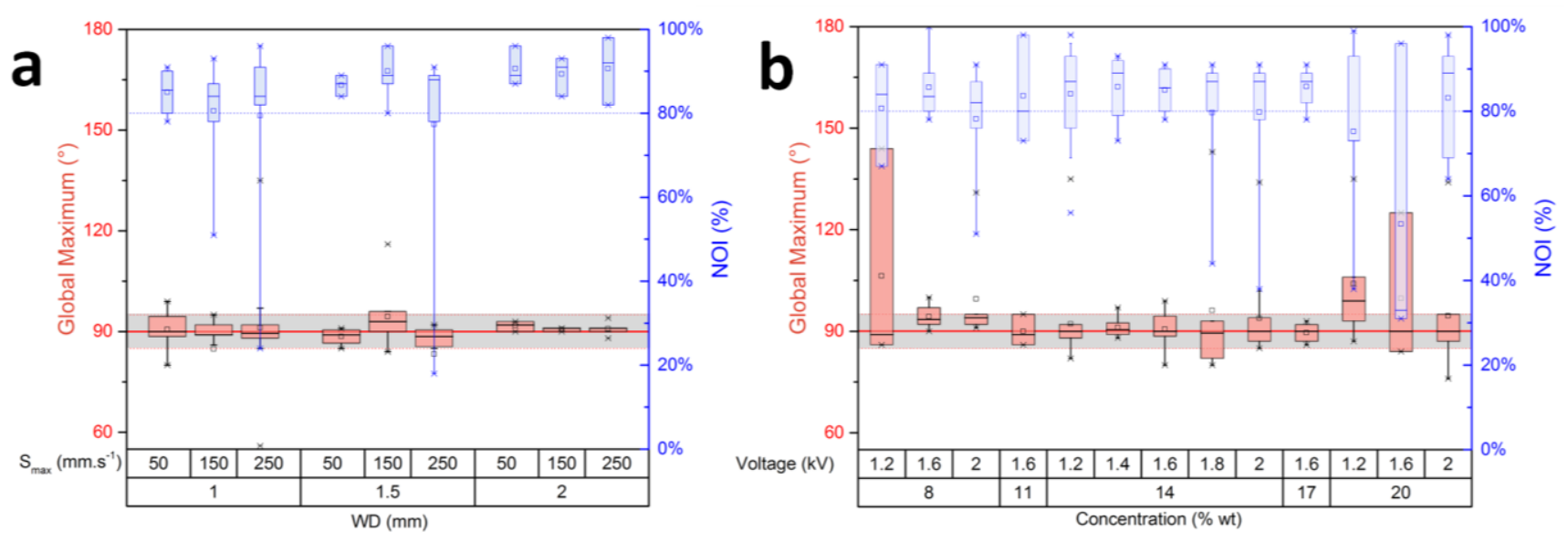

Figure 4. Orientation distribution statistics across varying process parameters. (a) Combined boxplots of the global maximum angle and NOI across samples generated by varying parameters of $S_{\max }$, and working distance. (b) Combined boxplots of the global maximum angle and NOI across samples generated by varying parameters of voltage and concentration. The dashed red lines bound the angles $85^{\circ}$ to $95^{\circ}$. The dashed blue lines indicate $80-100 \%$ NOI.

\subsubsection{Effect of process parameters on nanoarray fiber diameter}

The degree of success and conversely discontinuity in patterning is estimated in terms of the fibre density. We estimate the number of fibres in each sample by analysing microscope images. Fibres were first thinned to be one pixel in diameter, using DiameterJ, a plugin of ImageJ software. This applies both an axial thinning algorithm, developed by Zhang and Suen ${ }^{57}$, and a Voronoi tessellation algorithm, separately, then averages the result. By counting the pixels crossing four horizontal lines and taking the average, we estimate the number of fibres in the array. This was done semi-automatically using an ImageJ macro function.

Bowing during deposition can cause chaotic interactions with deposited fibers, resulting in entanglement as well as disruption to the continuity of deposition. The fiber must not be drawn beyond breaking point while minimizing the bowing of the fiber through more rapid drawing. The speeds of 50,150 and $250 \mathrm{~mm} \cdot \mathrm{s}^{-1}$ were found not to significantly influence the fibre number as predicted by this 
hypothetical breaking point disruption. This may be a case where critical breaking point speeds exceed the limitations of the system's max speed $\left(250 \mathrm{~mm} \cdot \mathrm{s}^{-1}\right)$. Fibre number for these parameters are shown in supplementary Figure 14.

Figure 5 (a-b) shows the mean diameter values for samples created in group T2 showing no clear trend with respect to concentration and voltage. Conventional electrospinning research has shown that the effect(s) of voltage, concentration, working distance and speed has a typically non-linear relationship with fibre diameter ${ }^{58,59}$, with the sensitivity of the output to process parameters described in work by Costolo et al. ${ }^{59}$. For the purposes of MTC fabrication, fiber diameter is preferably below ca. $700 \mathrm{~nm}$ 11,1213. Increased polymer concentration (see Figure 5 (a)) led to a pronounced variability in sample fibre diameter and variance, exhibiting the greatest population variance at $20 \%$ wt PEO in solution, with greatest mean diameter, $1.19 \pm 0.99 \mu \mathrm{m}$ across all parameters. From these results, samples made using $1.2 \mathrm{kV}$, at working distance of $1 \mathrm{~mm}$, at $\mathrm{S}_{\max } 50 \mathrm{~mm} \cdot \mathrm{s}^{-1}$ using $14 \mathrm{wt} \%$ solution were found to have the lowest mean diameter of $0.49 \pm 0.21 \mu \mathrm{m}$ (Figure 5 (b)), marginally lower than $8 \mathrm{wt} \%$ solution at $1.6 \mathrm{kV}$. Figure 5 (c) shows representative SEM micrographs of the fibers, each an example of the mean diameter for their associated voltage. For additional information on findings involving working distance diameter variation and fiber number refer to supplementary Figure 14. 

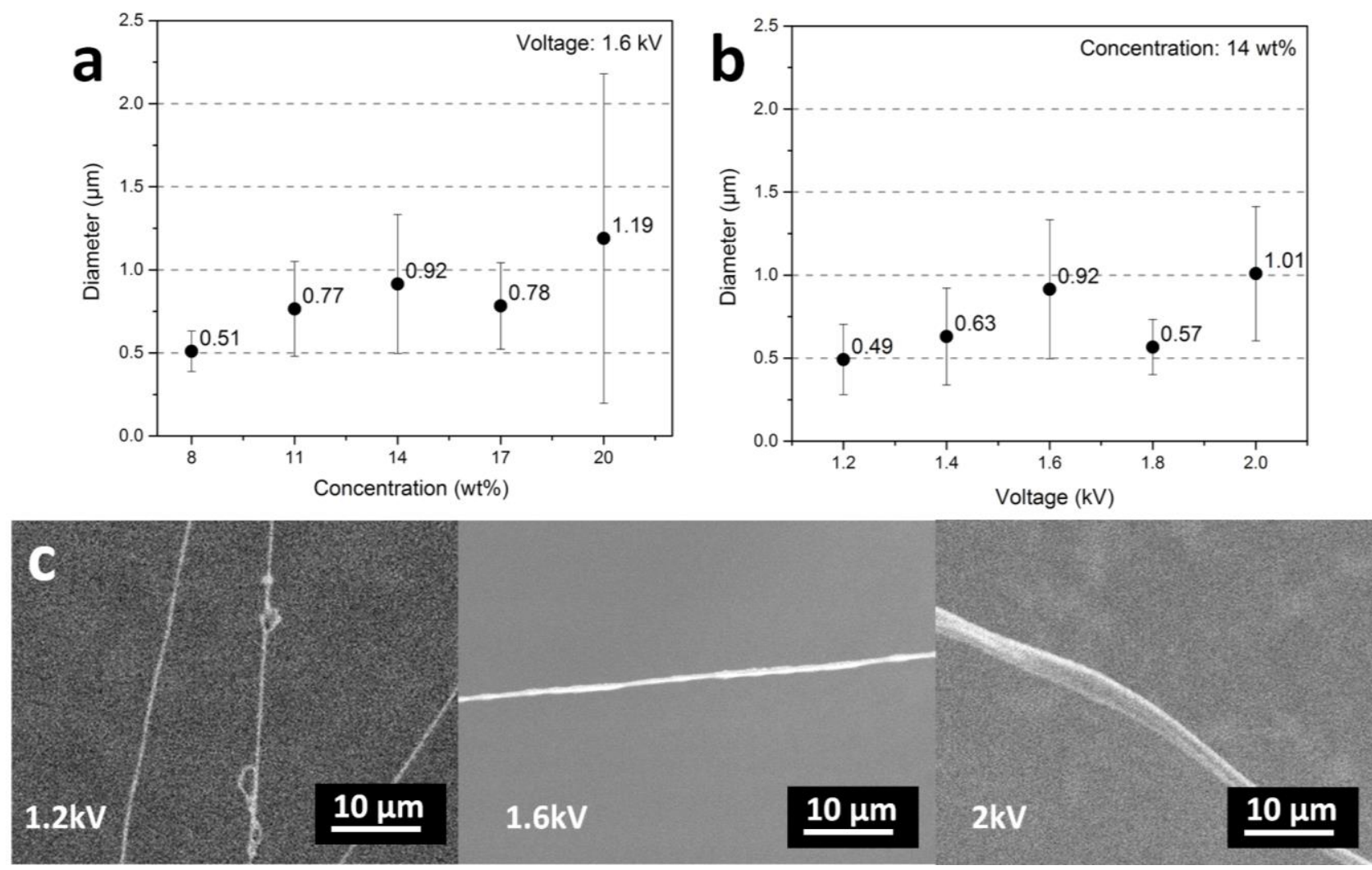

Figure 5. Fibre number and diameter as a function of process parameters. (a) Fibre diameter as a function of concentration and (b) voltage. (c) SEM images showing the morphology of fibres generated at different voltages. In the $2 \mathrm{kV}$ case, the image illustrates the surface morphology of the fiber, showing a twisted ribbon structure.

\subsection{Effect of pattern iteration on fibre number and diameter}

Fibrous mesh arrays were generated using the Si pattern, applying a range of iterations. Figure 6 (a) shows the nanostructure suspended between two pillar electrodes, produced using an iteration of 105 . The inset shows a magnified view of the fibres, showing an overlapping web structure. Figure 6 (b) shows the build-up of nanofiber at the corner of a pillar electrode. The stage must reduce speed in the corners of the $\mathrm{Si}$ tool-path in order to manoeuvre each pillar around the emitter tip. The disproportionate time the emitter is in proximity of these regions causes this build-up, producing complex striation structures shown magnified in figure 6 (c). 
A plot of the average fiber number as a function of iteration is shown in figure 6 (d), grouped by working distance. As the iteration value increases, we observe a phase exponential increase of the means in both working distance groups $(1 \mathrm{~mm}, 1.5 \mathrm{~mm})$. The data points indicated an exponential trend and fitting was further applied to determine the $\mathrm{R}^{2}$ coefficients. Fitted trends of the means were of the form: $\mathrm{y}=\mathrm{y}_{0}+\mathrm{Ae}^{-\mathrm{x} / \mathrm{t}}$, where $\mathrm{y}$ is the average fiber number, $\mathrm{x}$ is the iteration, $\mathrm{y}_{0}$ is the offset (or the plateau y value, the maximum fibers achievable at large iteration), $\mathrm{A}$ is the amplitude prefactor for the exponential, and $\mathrm{t}$ is the constant decay factor. The fittings achieved $\mathrm{R}^{2}$ values of $0.996(1.5 \mathrm{~mm}$ WD), and 0.998 (1 mm WD). $y_{0}$ is the plateau value, indicating the maximum fibers achievable based on this parameter setting. $\mathrm{t}$ is the decay factor and describes the 'speed' of decay, or the amount of iterations needed to reach the plateau of fibers, which relates to the inhibitory effect magnitude change with iteration. The relative change in fibre number declines at higher iterations. We attribute this effect to the established principle of decreasing electrospinning force as polymer mass at the collector increases ${ }^{32}$. This leads to increased inhibition of the electric field, lessening the drawing force on the polymer jet and inhibiting further ejection.

The suspended fibrous mesh shown in Figure 6 (e) was analysed for the fiber diameter using ImageJ plugin: DiameterJ. The normalised histogram of the fiber diameter in Figure 6 (e) is shown in Figure 6 (f), with a log-normal distribution fitting (red), peaking at ca. $0.2 \mu \mathrm{m}$. Figure 6 (g) shows the bar plot of mean diameter of each sample as a function of changing iteration and working distance. For working distance $1.5 \mathrm{~mm}$, the relative increase in fibre diameter and standard deviation at high iterations of 105 and 150 , can be explained by fibre overlapping, which generates bundled and fused fibers. This bundling combines several fibers (see supplementary section Figure 15) increasing the measured diameter as well as expanding the variability of fiber diameter, i.e. increasing the standard deviation. This effect is not seen in the $1 \mathrm{~mm}$ working distance samples, and it is not clear if there is any preventative effect or if bundling is simply a statistical occurrence 
Electric field in the SNFES process has a number of confounding effects. During patterning it varies in intensity and vector strength during the toolpath excursion. Supplementary section 5.4 and supplementary videos 2 and 3, shows the field change as the emitter is passed by the pillars in both Raster and Square iteration patterning. This was done with modelling software Comsol Multiphysics.

Further to this is that jetting fiber has some cascade effects on its successor and is affected by its predecessor as well. After much deposition, this too has an effect on fiber ejection, inhibiting and ultimately limiting the ejection of fiber.
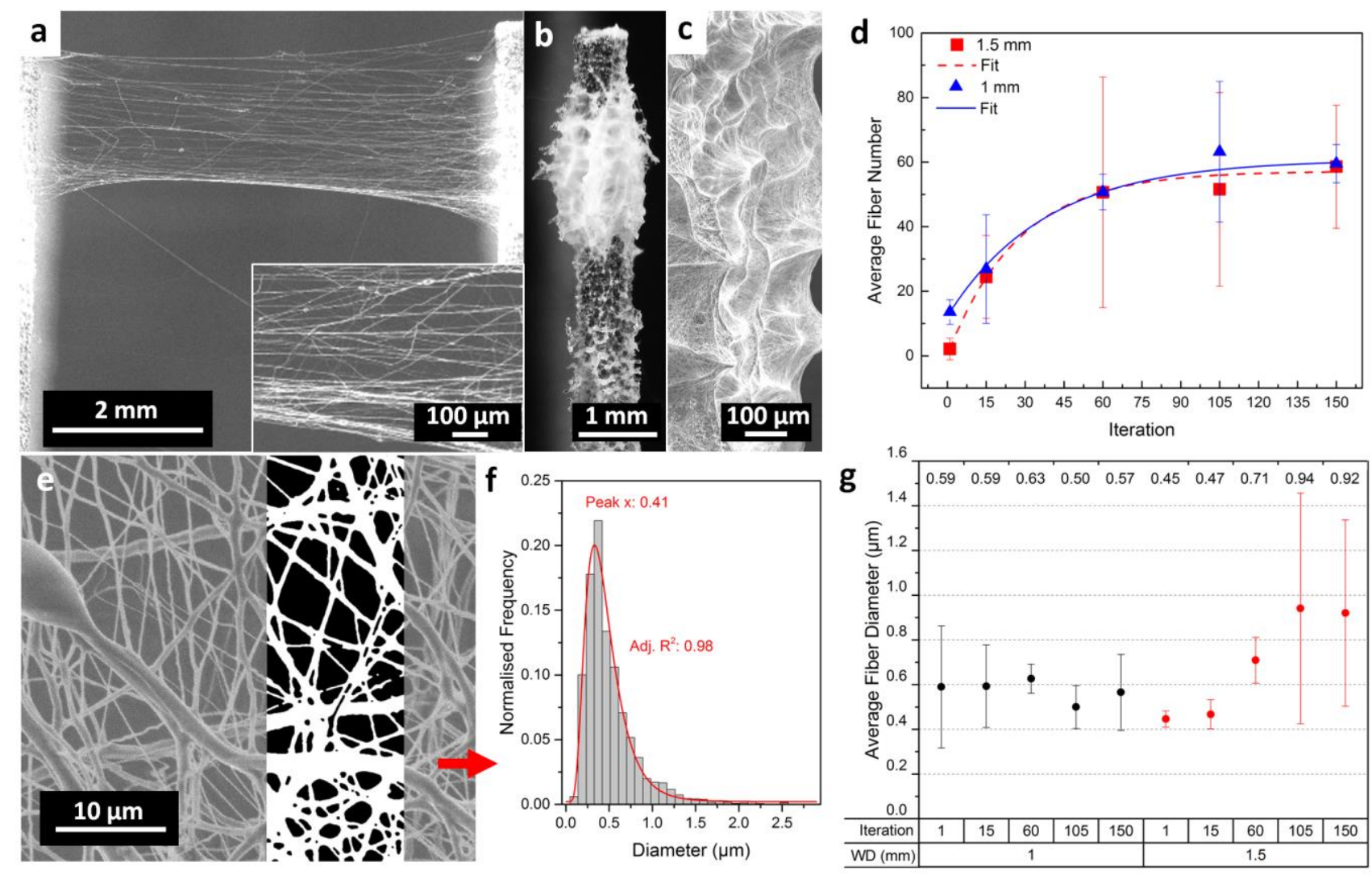

Figure 6. Iteration studies. (a) Microscopy of fibre array produced using 105 iterations, with high magnification inset. (b) Single electrode pillar showing fibrous build-up at the surface due to high iteration pattern deposition, with (c) SEM micrograph exhibiting the structure of the fibrous build-up. (d) Plot of the average fiber number as a function of iteration. Fitted trends of the means were of the form: $\mathrm{y}=\mathrm{y}_{0}+\mathrm{Ae}^{-\mathrm{x} / \mathrm{t}}$, where $\mathrm{y}$ is the average fiber number, $\mathrm{x}$ is the iteration, $\mathrm{y}_{0}$ is the offset (or the 
plateau y value at infinite $\mathrm{x}$ ), $\mathrm{A}$ is the amplitude prefactor for the exponential, and $\mathrm{t}$ is the constant decay factor. $\mathrm{R}^{2}$ values for each group: 0.996 (1.5 $\mathrm{mm} \mathrm{WD}$, red), and 0.998 (1 $\mathrm{mm} \mathrm{WD}$, blue). Error bars represent the s.d. of the means. (e) SEM micrograph of fibrous mesh, showing overlapping and semi-fused structure of repeated iterations (105 in this case). Segmented overlay shows the information that is input for image analysis. (f) Diameter frequency distribution for the micrograph (e). This distribution is fitted by a $\log$ normal distribution, with $\mathrm{R}^{2}$ value of 0.988 with centre of $0.41 \mu \mathrm{m} .(\mathrm{g})$ Plot of the distribution of fibre diameter as a function of iteration and working distance with point value $(\mu \mathrm{m})$ indicated in red.

\subsection{Pattern Variation}

\subsubsection{Effect of pattern and maximum speed on nanoarray distribution}

To demonstrate patterns of differing types, several pattern designs were developed as shown in figure, and this produced corresponding fibrous arrays as shown in figure. The electrodes produce a nonhomogeneous electric field, which the tip moves through while the jet changes direction based on the changing electric field intensity and direction (see supplementary Figure 12). Fabrication of patterns \, $V$ and $\backslash S i$ show direct control over fibre array distribution, within the four pillar volume. Figure 7 (a) shows a photo of the fibrous array produced using a Wi pattern. The nanoarrays are visible at this scale, due to the overlapping fibre density within the region. Figure 7 (b-d) shows top-down microscopy of the $\backslash, \backslash S i$, and $V$ patterned nano-arrays.

It should be noted that $V$ type patterning deviates from the intended outcome (see Figure 7 (d)), presenting a number of fibres spanning the exterior perimeter of the pillars similar to outcomes seen in Si pattern samples. This is despite the electrode motion never passing the perimeter directly. This implies that at some point fibers are drawn between these pillars without the influence mechanical drawing, solely through electrostatic attraction. This phenomenon may occur as a result of the diagonal 
pathing entering relatively higher proximity to tertiary pillars (e.g. at the midpoint of pathing) such that fiber jetting changes angle, hitting three separate pillars in transit along diagonal toolpaths, forming the $\mathrm{L}$ shape fiber array, instead of the intended two-point diagonal adjacent pillars. The statistical frequency may be managed in future through toolpath management in the form of arc pathing or through electrode design.

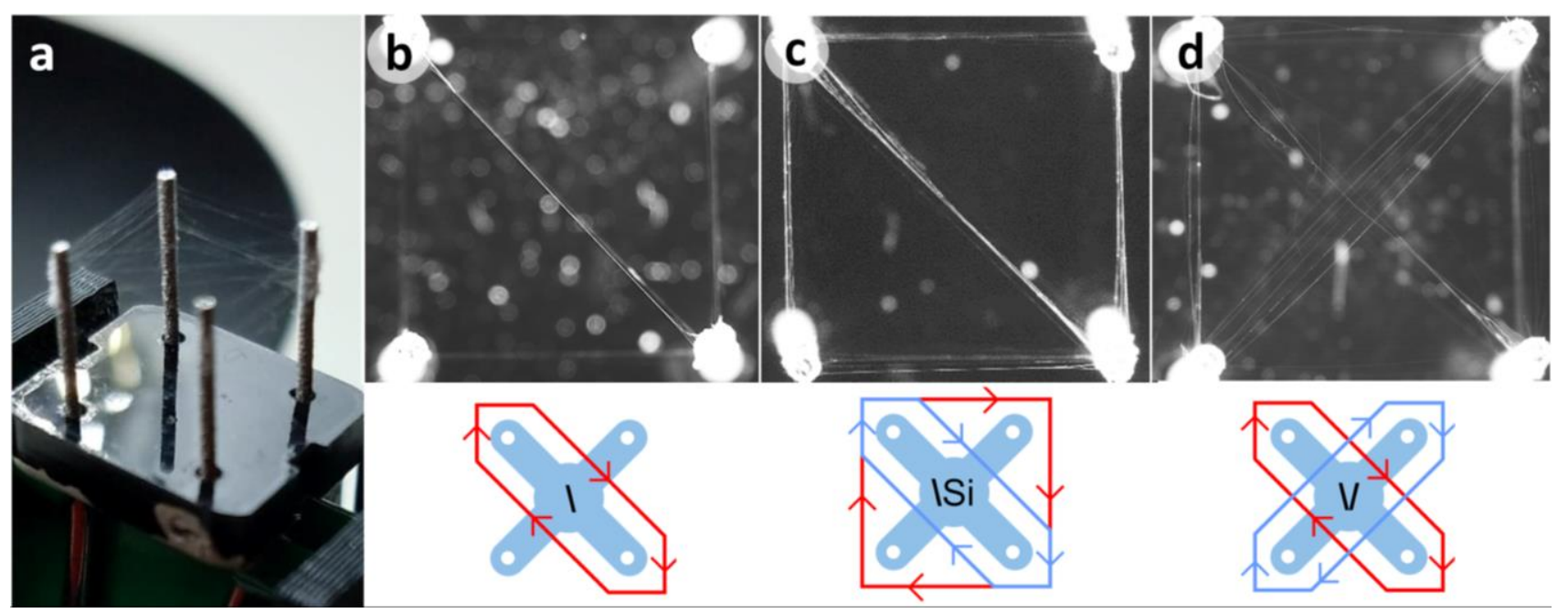

Figure 7. Pattern Variation. (a) Photograph of patterned nanofibers meshes suspended on electrode pillars. (b-d) Representative high magnification microscope images of PEO in three different patterns as indicated: toolpaths are indicated in alternate colours blue and red if separated events.

The histogram distribution of NOI for each pattern type is shown in Figure 8 (a). From the distributions we find the majority to be over $80 \%$ NOI, with the largest outliers in $\backslash$ pattern. This indicates reasonable variability of OD equivalent to the R patterns applying the same process parameters in section 3.1.1. Figure 8 (b) shows the distributions of global maximums between patterns as box plots, bounded in the region of $90 \pm 5^{\circ}$, indicating a precise outcome for each with high alignment. The ISi pattern (Figure 8 (c)) required move sequence consideration, given that the path can interfere with previously deposited fibers. A structure formed of Si followed by $\backslash$ would remove previously deposited fibres. 


\subsubsection{Effect of pattern and maximum speed on fibre density}

Figure 8 (c) shows the average number of fibres generated by each pattern. Inspection of Shapiro-Wilk normality test revealed that fibre number was normally distributed for all groups and by use of the Levene's test of homogeneity of variance, the variances were found to be significantly different. Univariate ANOVA was performed with the Games-Howell test comparing each group. It was found that, between patterns, the means were significantly different $(\mathrm{p}<0.01)$, with mean total fibre number in each group: $\backslash \mathrm{Si}(39 \pm 17.9), \mathrm{V}(22.8 \pm 6.9)$, and $\backslash(5.8 \pm 4.3)$. In contrast, changing the speed from 50 to $250 \mathrm{~mm} / \mathrm{s}^{-1}$ had a non-significant effect on the fibre number in each case $(\mathrm{p}>0.85)$. This result reinforces the previous finding that the maximum value of $250 \mathrm{~mm} \cdot \mathrm{s}^{-1}$ does not exceed a critical failure value, or causes significant discontinuities. Additionally, the change in pattern had no bearing on this effect. The differences in fiber number cannot be linked to the differing timeframes in which these patterns operate, as between 50 and $250 \mathrm{~mm} . \mathrm{s}-1$ as there is no significant change within each pattern group. The number is linked proportionally to the number of discrete moves (i.e. mechanical drawing operations) in each pattern. For example the $\backslash \mathrm{Si}$ pattern combines the $\backslash$ pattern and the Si pattern achieving a greater sum number of fibers due to the increased number of moves.
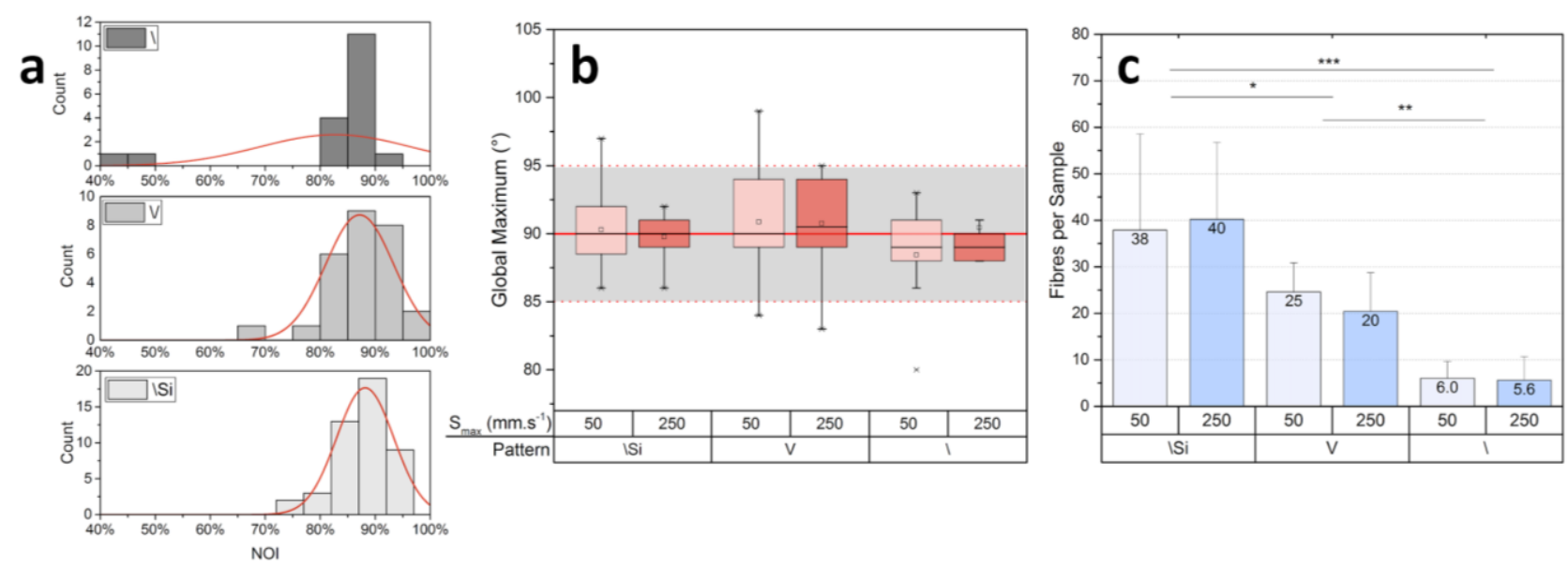

Figure 8. Pattern variation statistical information. (a) Normalised Orientation Index (NOI) histograms for each pattern type. (b) Box plots showing the distributions of the maximum peaks as a function of 
pattern and maximum speed. (c) Number of fibres per pattern type, as well as velocity. Data is presented as the mean \pm s.d. of four independent experiments $* \mathrm{P}<0.01$, ** $\mathrm{P}<0.001$, *** $\mathrm{P}<0.0001$; n.s., not significant. Significance was determined using Welch's t-test (two-sided).

\section{Conclusions}

Nanoarrays of PEO were patterned on 3D four-pillar electrodes, and analysed for their relative isotropy, alignment and diameter distribution. We first examined the process parameters for PEO by testing two process parameter groups: (i) max speed and working distance; (ii) voltage and concentration. In the majority of cases the alignment of the arrays was accurate to $\pm 5^{\circ}$. The exceptions were found in the extreme values of PEO concentration tested. The results of these experiments show that printed arrays can be significantly affected by the process parameters. An optimal setting from the findings suggest the most stable parameters for achieving a large number of submicron PEO fibers is $14 \mathrm{wt} \%, 1.4 \mathrm{kV}$, using the efficient 60 iterations at a WD of $1 \mathrm{~mm}$ with the Smax being the least influential parameter when applied in the range of 50-250 mm.s-1 .

In the second study, high density nanofiber meshes of PEO were patterned repeatedly, varying the number of iterations as well as maximum speed. The results showed a clear decaying exponential dependency of fibre number with iteration. The semi-automated NFES system was invaluable in the conduction of this repetitive process, as operations could take as long as two hours for high iteration value 150 , for example.

Finally several pattern types were investigated. These patterns exemplified the defining design space for arrays within the constraints of the electrodes used. The degree of control of array structure 
demonstrates the principle of this technique. The findings of this study are significant as it creates for the first time multiple suspended nanoarrays in a directed manner across multiple working planes in situ. This shows promise for future work which can utilize electrodes of greater complexity. Significantly the combination of additive metal SLM and automated instrument systems allowed the investigation of this suspension NFES technique.

This study contributes to the growing number of investigations into 3D culture and microtissue construct technologies. Suspended nanofiber arrays have high surface to volume ratio, potentially improved Young's modulus ${ }^{60}$, and can mimic the native ECM. Drug and neurotrophic factor release from fiber array scaffolds can achieve sustained, local factor delivery and contact guidance ${ }^{61}$. An example of the practical use of suspended fiber arrays is given in work by Kriebel et al. ${ }^{62}$, producing a nerve guide construct containing PCL arrays. This research builds on our previous work ${ }^{4}$, creating more complex patterns, developed for potential contact guidance and drug delivery applications. This technique is in its earliest development. Further studies, examining the feasibility of SNFES for the above applications, are underway. Ongoing studies aimed at integrating nanoarrays within 3D hydrogel constructs, will rely on improving the precision of the SNFES method.

This work highlights the potential of this new approach as a means of developing 3D scaffold nanostructures large enough for use in microtissue constructs for the medical research sector. Future work will aim to explore the use of biopolymers as well as biodegradable polymers (e.g. polyesters) to address cell culture and tissue engineering applications. This new technique adds a further dimension in nanofabrication tools, allowing the construction of consistent nanofibrous MTCs in a manner that facilitates cell culture, and tissue engineering studies, presenting an opportunity to examine the effects of nanofiber arrays distribution in 3D cell culture. 


\subsection{Acknowledgements}

The authors acknowledge the financial support of the Australian Research Council (ARC) Centre of Excellence Scheme (Project CE 140100012). The authors thank the Australian National Fabrication Facility (ANFF) for providing facilities for the fabrication of the NFES system components. The authors thank the University of Wollongong Electron Microscopy Centre (EMC) for providing SEM facilities. The authors thank Mr. Grant Barnsley for his support in the fabrication of collector electrode arrays, and Mr Benjamin Filipi for his work in the production of the automatic cleaning device.

\subsection{References}

1. Cullen, D. K. et al. Microtissue engineered constructs with living axons for targeted nervous system reconstruction. Tissue Eng. Part A 18, 2280-2289 (2012).

2. Schrobback, K., Klein, T. J. \& Woodfield, T. B. F. The importance of connexin hemichannels during chondroprogenitor cell differentiation in hydrogel versus microtissue culture models. Tissue Eng. Part A 21, 1785-1794 (2015).

3. Legant, W. R., Chen, C. S. \& Vogel, V. Force-induced fibronectin assembly and matrix remodeling in a 3D microtissue model of tissue morphogenesis. Integr. Biol. 4, 1164-1174 (2012).

4. Zhang, B. G. X. et al. Recent advances in nerve tissue engineering. Int. J. Artif. Organs 37, 277-291 (2014).

5. Vieira, M. S. et al. Neural stem cell differentiation into mature neurons: Mechanisms of regulation and biotechnological applications. Biotechnol. Adv. 36, 1946-1970 (2018).

6. Flemming, R. G., Murphy, C. J., Abrams, G. A., Goodman, S. L. \& Nealey, P. F. Effects of synthetic micro- and nano-structured surfaces on cell behavior. Biomaterials 20, 573-588 (1999).

7. Abrams, G. A., Schaus, S. S., Goodman, S. L., Nealey, P. F. \& Murphy, C. J. Nanoscale topography of the corneal epithelial basement membrane and Descemet's membrane of the human. Cornea 19, 57-64 (2000).

8. Morrison, S. J. \& Spradling, A. C. Stem cells and niches: mechanisms that promote stem cell 
maintenance throughout life. Cell 132, 598-611 (2008).

9. Semino, C. E., Merok, J. R., Crane, G. G., Panagiotakos, G. \& Zhang, S. Functional differentiation of hepatocyte-like spheroid structures from putative liver progenitor cells in three-dimensional peptide scaffolds. Differentiation 71, 262-270 (2003).

10. Wolf, K. et al. Compensation mechanism in tumor cell migration: mesenchymal-amoeboid transition after blocking of pericellular proteolysis. J. Cell Biol. 160, 267-277 (2003).

11. Yang, F., Murugan, R., Wang, S. \& Ramakrishna, S. Electrospinning of nano/micro scale poly(L-lactic acid) aligned fibers and their potential in neural tissue engineering. Biomaterials 26, 2603-2610 (2005).

12. Kijeńska, E., Prabhakaran, M. P., Swieszkowski, W., Kurzydlowski, K. J. \& Ramakrishna, S. Interaction of Schwann cells with laminin encapsulated PLCL core-shell nanofibers for nerve tissue engineering. Eur. Polym. J. 50, 30-38 (2014).

13. Beachley, V. \& Wen, X. Polymer nanofibrous structures: Fabrication, biofunctionalization, and cell interactions. Prog. Polym. Sci. 35, 868-892 (2010).

14. Yao, L., O’Brien, N., Windebank, A. \& Pandit, A. Orienting neurite growth in electrospun fibrous neural conduits. J. Biomed. Mater. Res. B Appl. Biomater. 90, 483-491 (2009).

15. Quigley, A. F. et al. A conducting-polymer platform with biodegradable fibers for stimulation and guidance of axonal growth. Adv. Mater. 21, 4393-4397 (2009).

16. Liu, X. et al. Guidance of neurite outgrowth on aligned electrospun polypyrrole/poly(styrene-betaisobutylene-beta-styrene) fiber platforms. J. Biomed. Mater. Res. A 94, 1004-1011 (2010).

17. Teo, W. E. \& Ramakrishna, S. Electrospun fibre bundle made of aligned nanofibres over two fixed points. Nanotechnology 16, 1878-1884 (2005).

18. Pham, Q. P., Sharma, U. \& Mikos, A. G. Electrospun Poly( $\varepsilon$-caprolactone) Microfiber and Multilayer Nanofiber/Microfiber Scaffolds: Characterization of Scaffolds and Measurement of Cellular Infiltration. Biomacromolecules 7, 2796-2805 (2006).

19. Prabhakaran, M. P., Venugopal, J., Chan, C. K. \& Ramakrishna, S. Surface modified electrospun nanofibrous scaffolds for nerve tissue engineering. Nanotechnology 19, 455102 (2008).

20. Vasita, R. \& Katti, D. S. Nanofibers and their applications in tissue engineering. Int. J. Nanomedicine 1, 15-30 (2006). 
21. Neal, R. A. et al. Alignment and composition of laminin-polycaprolactone nanofiber blends enhance peripheral nerve regeneration. J. Biomed. Mater. Res. A 100, 406-423 (2012).

22. He, J.-H., Liu, Y. \& Xu, L. Apparatus for preparing electrospun nanofibres: a comparative review. Mater. Sci. Technol. 26, 1275-1287 (2010).

23. Khan, W. S., Asmatulu, R., Ceylan, M. \& Jabbarnia, A. Recent progress on conventional and nonconventional electrospinning processes. Fibers Polym. 14, 1235-1247 (2013).

24. Teo, W.-E., Inai, R. \& Ramakrishna, S. Technological advances in electrospinning of nanofibers. Sci. Technol. Adv. Mater. 12, 013002 (2011).

25. Yang, F., Xu, C. Y., Kotaki, M., Wang, S. \& Ramakrishna, S. Characterization of neural stem cells on electrospun poly(L-lactic acid) nanofibrous scaffold. J. Biomater. Sci. Polym. Ed. 15, 1483-1497 (2004).

26. Agarwal, S., Wendorff, J. H. \& Greiner, A. Progress in the field of electrospinning for tissue engineering applications. Adv. Mater. 21, 3343-3351 (2009).

27. Bajgai, M. P. et al. Poly( $\varepsilon$-caprolactone) grafted dextran biodegradable electrospun matrix: A novel scaffold for tissue engineering. J. Appl. Polym. Sci. 108, 1447-1454 (2008).

28. Zhu, Y., Cao, Y., Pan, J. \& Liu, Y. Macro-alignment of electrospun fibers for vascular tissue engineering. J. Biomed. Mater. Res. B Appl. Biomater. 92, 508-516 (2010).

29. Stitzel, J. et al. Controlled fabrication of a biological vascular substitute. Biomaterials 27, 1088-1094 (2006).

30. Yixiang, D., Yong, T., Liao, S., Chan, C. K. \& Ramakrishna, S. Degradation of electrospun nanofiber scaffold by short wave length ultraviolet radiation treatment and its potential applications in tissue engineering. Tissue Eng. Part A 14, 1321-1329 (2008).

31. He, W., Ma, Z., Yong, T., Teo, W. E. \& Ramakrishna, S. Fabrication of collagen-coated biodegradable polymer nanofiber mesh and its potential for endothelial cells growth. Biomaterials 26, 7606-7615 (2005).

32. Doshi, J. \& Reneker, D. H. Electrospinning process and applications of electrospun fibers. J. Electrostat. 35, 151-160 (1995).

33. Wei, J. et al. Electrohydrodynamic Direct Writing Platform Based on Near-Field Electrospinning. Key Eng. Mater. 562-565, 614-619 (2013). 
34. Huang, Y. et al. Versatile, kinetically controlled, high precision electrohydrodynamic writing of micro/nanofibers. Sci. Rep. 4, 5949 (2014).

35. He, J., Xu, F., Dong, R., Guo, B. \& Li, D. Electrohydrodynamic 3D printing of microscale poly ( $\varepsilon-$ caprolactone) scaffolds with multi-walled carbon nanotubes. Biofabrication 9, 015007 (2017).

36. Hochleitner, G. et al. Additive manufacturing of scaffolds with sub-micron filaments via melt electrospinning writing. Biofabrication 7, 035002 (2015).

37. Lee, M. \& Kim, H.-Y. Toward nanoscale three-dimensional printing: nanowalls built of electrospun nanofibers. Langmuir 30, 1210-1214 (2014).

38. Luo, G. et al. Direct-Write, Self-Aligned Electrospinning on Paper for Controllable Fabrication of ThreeDimensional Structures. ACS Appl. Mater. Interfaces 7, 27765-27770 (2015).

39. Luo, G. et al. High aspect-ratio 3D microstructures via near-field electrospinning for energy storage applications. in 2016 IEEE 29th International Conference on Micro Electro Mechanical Systems (MEMS) (2016). doi:10.1109/memsys.2016.7421549

40. Visser, J. et al. Reinforcement of hydrogels using three-dimensionally printed microfibres. Nat. Commun. 6, 6933 (2015).

41. Hochleitner, G. et al. Additive manufacturing of scaffolds with sub-micron filaments via melt electrospinning writing. Biofabrication 7, 035002 (2015).

42. Visser, J. et al. Reinforcement of hydrogels using three-dimensionally printed microfibres. Nat. Commun. 6, 6933 (2015).

43. Fuh, Y.-K., Wu, Y.-C., He, Z.-Y., Huang, Z.-M. \& Hu, W.-W. The control of cell orientation using biodegradable alginate fibers fabricated by near-field electrospinning. Mater. Sci. Eng. C Mater. Biol. Appl. 62, 879-887 (2016).

44. Nagle, A. R. et al. A Direct 3D Suspension Near-Field Electrospinning Technique for the Fabrication of Polymer Nanoarrays. Nanotechnology (2019). doi:10.1088/1361-6528/ab011b

45. Martinez-Prieto, N. et al. Feasibility of Fiber-Deposition Control by Secondary Electric Fields in NearField Electrospinning. Journal of Micro and Nano-Manufacturing 3, 041005 (2015).

46. Chang, C., Limkrailassiri, K. \& Lin, L. Continuous near-field electrospinning for large area deposition of orderly nanofiber patterns. Appl. Phys. Lett. 93, 123111 (2008). 
47. Hotaling, N. A., Bharti, K., Kriel, H. \& Simon, C. G., Jr. DiameterJ: A validated open source nanofiber diameter measurement tool. Biomaterials 61, 327-338 (2015).

48. Rezakhaniha, R. et al. Experimental investigation of collagen waviness and orientation in the arterial adventitia using confocal laser scanning microscopy. Biomech. Model. Mechanobiol. 11, 461-473 (2011).

49. Sacks, M. S., Smith, D. B. \& Hiester, E. D. A small angle light scattering device for planar connective tissue microstructural analysis. Ann. Biomed. Eng. 25, 678-689 (1997).

50. D’Amore, A., Stella, J. A., Wagner, W. R. \& Sacks, M. S. Characterization of the complete fiber network topology of planar fibrous tissues and scaffolds. Biomaterials 31, 5345-5354 (2010).

51. Tutak, W. et al. The support of bone marrow stromal cell differentiation by airbrushed nanofiber scaffolds. Biomaterials 34, 2389-2398 (2013).

52. Angammana, C. J. \& Jayaram, S. H. Analysis of the Effects of Solution Conductivity on Electrospinning Process and Fiber Morphology. IEEE Trans. Ind. Appl. 47, 1109-1117 (2011).

53. Liu, Y. et al. Effects of solution properties and electric field on the electrospinning of hyaluronic acid. Carbohydr. Polym. 83, 1011-1015 (2011).

54. Jaworek, A. \& Sobczyk, A. T. Electrospraying route to nanotechnology: An overview. J. Electrostat. 66, $197-219$ (2008).

55. Liu, J. et al. Bead-on-string structure printed by electrohydrodynamic jet under alternating current electric field. Appl. Phys. A: Mater. Sci. Process. 122, (2016).

56. Phung, T. H., Kim, S. \& Kwon, K.-S. A high speed electrohydrodynamic (EHD) jet printing method for line printing. J. Micromech. Microeng. 27, 095003 (2017).

57. Zhang, T. Y. \& Suen, C. Y. A fast parallel algorithm for thinning digital patterns. Commun. ACM 27, 236-239 (1984).

58. Ghorani, B. \& Tucker, N. Fundamentals of electrospinning as a novel delivery vehicle for bioactive compounds in food nanotechnology. Food Hydrocoll. 51, 227-240 (2015).

59. Costolo, M. A., Lennhoff, J. D., Pawle, R., Rietman, E. A. \& Stevens, A. E. A nonlinear system model for electrospinning sub-100 nm polyacrylonitrile fibres. Nanotechnology 19, 035707 (2008).

60. Yao, J., Bastiaansen, C. \& Peijs, T. High Strength and High Modulus Electrospun Nanofibers. Fibers 2 , 
$158-186$ (2014).

61. Beachley, V., Katsanevakis, E., Zhang, N. \& Wen, X. A Novel Method to Precisely Assemble Loose Nanofiber Structures for Regenerative Medicine Applications. Advanced Healthcare Materials 2, 343351 (2013).

62. Kriebel, A. et al. Three-dimensional configuration of orientated fibers as guidance structures for cell migration and axonal growth. J. Biomed. Mater. Res. B Appl. Biomater. 102, 356-365 (2014). 